:::: Helmholtz-Zentrum

$\because \because:$ Geesthacht

Zentrum für Material- und Küstenforschung

\title{
Original
}

Graf, G.; Drescher, S.; Meister, A.; Haramus, V.M.; Dobner, B.; Blume, A.:

Tuning the aggregation behaviour of single-chain bolaamphiphiles in aqueous suspension by changes in headgroup asymmetry

In: Soft Matter (2013) Royal Society of Chemistry

DOI: 10.1039/C3SM51778K 
Cite this: Soft Matter, 2013, 9, 9562

Received 28th June 2013

Accepted 12th August 2013

DOI: $10.1039 / c 3 s m 51778 k$

www.rsc.org/softmatter

\section{Tuning the aggregation behaviour of single-chain bolaamphiphiles in aqueous suspension by changes in headgroup asymmetryं}

\author{
Gesche Graf, ${ }^{a}$ Simon Drescher, ${ }^{\text {ab }}$ Annette Meister, ${ }^{\text {ac }}$ Vasil M. Garamus, ${ }^{d}$ Bodo Dobner $^{\mathrm{e}}$ \\ and Alfred Blume*a
}

\begin{abstract}
The self-assembly process in aqueous suspension of two new asymmetrical single-chain bolaamphiphiles, namely 32-\{[hydroxy(2-hydroxyethoxy)phosphinyl]oxy\}dotriacontane-1-yl-\{2-[N-(3-dimethylaminopropyl)$N, N$-dimethylammonio]ethylphosphate (DMAPPC-C32-POH) and 32-hydroxydotriacontane-1-yl- $\{2-[N$-(3dimethylaminopropyl)- $\mathrm{N}, \mathrm{N}$-dimethylammonio]ethylphosphate\} (DMAPPC-C32-OH), was studied as a function of temperature using transmission electron microscopy, differential scanning calorimetry, FT-IRspectroscopy, small angle neutron and small angle X-ray scattering to determine whether the asymmetry of the molecule induces the formation of types of aggregates other than the well characterized helical nanofibres of structurally similar symmetrical single-chain bolaamphiphiles with identical headgroups. DMAPPC-C32-POH in acetate buffer at $\mathrm{pH} 5$ can still form nanofibres, i.e. the asymmetry does not induce the formation of other aggregate structures. However, the fibres display a tendency to break more easily and to form irregular, circular structures. This is also reflected by the rheological properties of the suspension that reveal decreased strain resistance at $\mathrm{pH} 5$. In aqueous suspensions at $\mathrm{pH} 10$, where the headgroups of the molecule are negatively charged, only short fibre segments are formed and no gel formation occurs. At higher temperature these fibres convert into micellar aggregates as observed before for symmetrical bolalipids with large headgroups. In contrast, in aqueous suspensions of DMAPPC-C32-OH, a bolalipid where the size difference of the headgroups is much larger, lamellar structures are formed at $\mathrm{pH} 10$ where the headgroup of the molecule is zwitterionic. At low temperature, the molecules are packed in an orthorhombic lattice with interdigitated chains and a repeat distance between lamellae of $6.2 \mathrm{~nm}$ is observed. An increase in temperature leads to a lamellar phase with hexagonal packing of the chains. The chains become liquidcrystalline only at very high temperature above $90^{\circ} \mathrm{C}$. At low $\mathrm{pH}$, when the headgroup of the molecule becomes positively charged, some short elongated micellar aggregates are seen besides sheet-like structures. A temperature increase leads to a similar sequence of transformations of the chain packing until formation of a liquid-crystalline lamellar phase at a temperature close to $90^{\circ} \mathrm{C}$. The results show that the aggregation behaviour of single-chain bolaamphiphiles can not only be tuned by changes in chain length or size of both headgroups but also by the difference in headgroup size and charge in asymmetric bolaamphiphiles.
\end{abstract}

\section{Introduction}

anstitute of Chemistry, Physical Chemistry, MLU Halle-Wittenberg, Von-Danckelmann-Platz 4, 06120 Halle (Saale), Germany

${ }^{b}$ Institute of Pharmacy, Pharmaceutical Chemistry, MLU Halle-Wittenberg, WolfgangLangenbeck-Str. 4, 06120 Halle (Saale), Germany

${ }^{c}$ Mitteldeutsches Zentrum für Struktur und Dynamik der Proteine, Biocenter, MLU Halle-Wittenberg, Weinbergweg 22, 06120 Halle (Saale), Germany

${ }^{d}$ Helmholtz-Zentrum Geesthacht: Zentrum für Material- und Küstenforschung GmbH, Max-Planck-Str. 1, 21502 Geesthacht, Germany

${ }^{e}$ Institute of Pharmacy, Biochemical Pharmacy, MLU Halle-Wittenberg, WolfgangLangenbeck-Str. 4, 06120 Halle (Saale), Germany. E-mail: alfred.blume@chemie. uni-halle.de

$\dagger$ Electronic supplementary information (ESI) available: Detailed FT-IR and rheological measurements. See DOI: 10.1039/c3sm51778k
Symmetrical bolaamphiphiles with a single alkyl chain connecting two large polar groups self-assemble into helical nanofibres formed by molecules with all-trans alkyl chains. These nanofibres gel water in aqueous suspensions by creating a three-dimensional network. ${ }^{1-8}$ By varying the molecular structure of the bolaamphiphiles the aggregation properties can be influenced and controlled in a desired way. To realize this goal changes in either the headgroup region or in the spacer chain of the molecules can be introduced. ${ }^{8}$ For instance, differently sized identical headgroups can be coupled to both ends, ${ }^{\text {9,10 }}$ the alkyl chain length can be decreased or 
increased ${ }^{11-13}$ or heteroatoms can be incorporated into the spacer chain., ${ }^{9,14}$

A further possibility of modifying the structure leading to changes in aggregation properties is the coupling of two different headgroups to the same alkyl chain. Due to two headgroups with different sizes the formation of lamellar aggregates, ${ }^{15-17}$ e.g., with antiparallel orientation of the different headgroups, or rods and nanotubes ${ }^{16,18}$ as described for other asymmetrical bolaamphiphiles can be favoured. In these cases, the size difference between the two headgroups has to be such that the molecules fit into either a monolayer-like structure for the lamellar aggregates with antiparallel orientation of the molecules or into the different curvature of the inner and outer side of the nanotubes.

Starting from symmetrical bolaamphiphiles with two large hydrophilic headgroups, the question arose, whether the size difference of the two headgroups of the asymmetrical bolaamphiphile DMAPPC-C32-POH ${ }^{19}$ (32-\{[hydroxy(2-hydroxyethoxy)phosphinyl]oxy\}dotriacontane-1-yl-\{2-[N-(3-dimethylaminopropyl)$\mathrm{N}, \mathrm{N}$-dimethylammonio]ethylphosphate\}) is large enough to result in significant changes of the aggregation behaviour in aqueous suspension. The asymmetrical bolaamphiphile DMAPPC-C32$\mathrm{POH}$ consists of two different headgroups at each end of an alkyl chain with 32 methylene units. The chemical structure of this molecule is shown in Fig. 1. The molecule contains functional groups that can be protonated or deprotonated depending on the $\mathrm{pH}$ value of the aqueous solution.

A second bolaamphiphile with an even larger size difference of both headgroups was also synthesized. ${ }^{19}$ The compound DMAPPC-C32-OH (32-hydroxydotriacontane-1-yl-\{2-[N-(3-dimethylaminopropyl)- $N, N$-dimethylammonio]ethylphosphate\}) (see Fig. 1) has on one side the same polar group as DMAPPC-C32$\mathrm{POH}$, but on the other side a much smaller headgroup, namely just a hydroxyl group.

The aggregation behaviour in aqueous suspension of these two new asymmetrical bolaamphiphiles is expected to be influenced not only by the size difference of the two headgroups but also by their charge state. This can be influenced by changing the $\mathrm{pH}$ of the aqueous buffer. We therefore investigated the aggregation behaviour of these bolalipids in suspensions of different pH-values using differential scanning calorimetry (DSC), Fourier-transform infrared spectroscopy (FTIR), transmission electron microscopy (TEM), small angle neutron scattering (SANS), small angle X-ray scattering (SAXS), and rheological measurements. We will show that by tuning the size difference as well as the charge of the headgroups different aggregates will be formed.

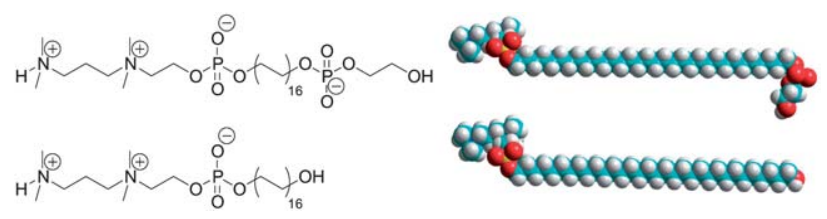

Fig. 1 Chemical structures and CPK-models of (top) DMAPPC-C32-POH and (bottom) DMAPPC-C32-OH with protonated amino groups (at $\mathrm{pH}$ 5).

\section{Experimental}

\subsection{Materials}

Solvents and buffer salts were purchased from Carl Roth $\mathrm{GmbH}$ \& Co. KG (Karlsruhe, Germany) and used as received. Ultra-pure water was used from a Millipore Milli-Q A10 system (Millipore $\mathrm{GmbH}$, Schwalbach, Germany). Both bolaamphiphiles, 32$\{[$ hydroxy(2-hydroxyethoxy)phosphinyl]oxy\}dotriacontane-1-yl$\{2$-[N-(3-dimethylaminopropyl)- $N, N$-dimethylammonio]ethylphosphate (DMAPPC-C32-POH) and 32-hydroxydotriacontane1-yl- $\{2-[N$-(3-dimethylaminopropyl)- $N, N$-dimethylammonio]ethylphosphate\} (DMAPPC-C32-OH) were synthesized according to procedures which are described separately. ${ }^{19}$

\subsection{Sample preparation}

The appropriate amount of bolaamphiphile was suspended in acetate buffer (10 mM, pH 5), or carbonate buffer $(10 \mathrm{mM}, \mathrm{pH}$ 10), respectively. To achieve a homogeneous suspension the samples were heated above $70{ }^{\circ} \mathrm{C}$ three times and vortexed.

\subsection{Differential scanning calorimetry (DSC)}

Measurements were carried out with a MicroCal VP-DSC (MicroCal Inc., Northampton, USA). The bolaamphiphiles were suspended in acetate buffer (10 mM, pH 5) or carbonate buffer (10 mM, pH 10) at a concentration of $1 \mathrm{mg} \mathrm{ml}^{-1}$, and the same buffer was used as the reference. Measurements were carried out using heating rates of $20{ }^{\circ} \mathrm{C} \mathrm{min}{ }^{-1}$ in the temperature interval from 2 to $95{ }^{\circ} \mathrm{C}$. To check the reproducibility at least three consecutive heating and cooling scans were recorded for each sample. The buffer-buffer baseline was subtracted from the thermograms of the samples, and the DSC scans were evaluated using Origin 8.0 software.

\subsection{Fourier-transform infrared spectroscopy (FT-IR)}

FT-IR-spectra as a function of temperature were recorded with a Bruker Vector 22 Fourier-transform spectrometer (Bruker Optik $\mathrm{GmbH}$, Karlsruhe, Germany) operating at $2 \mathrm{~cm}^{-1}$ resolution. The bolaamphiphile suspension $\left(c=50 \mathrm{mg} \mathrm{ml}^{-1}\right)$ in acetate buffer (300 mM, pH 5) or carbonate buffer (300 mM, pH 10) was placed between two $\mathrm{CaF}_{2}$ windows separated with a $12 \mu \mathrm{m}$ Teflon spacer and equilibrated for $2 \mathrm{~h}$ at $5{ }^{\circ} \mathrm{C}$ prior to the measurement. 64 scans each were recorded in the temperature range from 5 to $95{ }^{\circ} \mathrm{C}$ with an equilibration time of $8 \mathrm{~min}$ at each temperature. Spectra of buffer were measured with an identical setup and were subtracted from the sample spectra using the OPUS software supplied by Bruker Optik GmbH.

\subsection{Transmission electron microscopy (TEM)}

TEM images were recorded with a Zeiss EM 900 transmission electron microscope (Carl Zeiss GmbH, Oberkochen, Germany). $5 \mu \mathrm{l}$ of the sample solution $\left(c=0.3-0.1 \mathrm{mg} \mathrm{ml}^{-1}\right)$ was spread on a copper grid coated with a Formvar film. After 1 minute the excess solution was blotted off with a filter paper. The samples were stained with $1 \%$ uranyl acetate $(20 \mu \mathrm{l})$ solution, which was 
drained off after 1 minute. The samples were dried overnight at room temperature.

\subsection{Small angle neutron scattering (SANS)}

Experiments were carried out with the SANS-1 instrument at the FRG 1 research reactor, Helmholtz Zentrum Geesthacht, Germany. The bolaamphiphiles were suspended in deuterated acetate puffer at pH $5(10 \mathrm{mM})$ at a concentration of $1 \mathrm{mg} \mathrm{ml} \mathrm{m}^{-1}$ and the suspensions were filtered through a membrane filter of $0.45 \mu \mathrm{m}$ pore size at $80{ }^{\circ} \mathrm{C}$. The samples were filled into quartz cuvettes with a path length of $5 \mathrm{~mm}$ and inserted into a thermostatted sample holder $\left(\Delta T=0.5^{\circ} \mathrm{C}\right)$. Four sample-to-detector distances were employed to cover the range of scattering vectors $q$ from 0.05 to $2.5 \mathrm{~nm}^{-1}$. Transmission of the samples was approx. $70 \%$ and the contribution of multiple scattering could be neglected. The raw scattering data were corrected for the background scattering from the solvent, sample cell, and other sources using conventional procedures. Subsequently, the scattering data were fitted using the indirect Fourier transform method..$^{2021}$ A detailed description of this process was given by Meister et al. ${ }^{12,22}$

\subsection{Small angle X-ray scattering (SAXS)}

The SAXS measurements were performed with a laboratory SAXS instrument (Nanostar, Bruker AXS GmbH, Karlsruhe, Germany). The instrument includes an I $\mu \mathrm{S}$ micro-focus X-ray source with a power of $30 \mathrm{~W}$ using the wavelength of the $\mathrm{Cu} \mathrm{K} \alpha$ line. As the detector, a VÅNTEC-2000 detector $\left(14 \times 14 \mathrm{~cm}^{2}\right.$ and $2048 \times 2048$ pixels) was used. The sample to detector distance was $108.3 \mathrm{~cm}$ and the accessible $q$ range was 0.1 to $2.3 \mathrm{~nm}^{-1}$. Samples were filled into glass capillaries of $2 \mathrm{~mm}$ diameter. The raw scattering data were corrected for the background from the solvent measured in a capillary with the same diameter and then converted to absolute units using the scattering of pure water measured at $20^{\circ} \mathrm{C}$ (program SuperSAXS, Prof. C. L. P. de Oliveira and Prof. J. S. Pedersen).

\subsection{Rheology}

Oscillatory rheological measurements were carried out with an Anton Paar MCR 301 Rheometer (Anton Paar GmbH, Graz, Austria) controlled by the software Rheoplus 3.0. A cone-plate shear system $\left(2^{\circ}, 50 \mathrm{~mm}\right)$ with a thermostatting unit (Peltier system: $-40{ }^{\circ} \mathrm{C}$ to $200{ }^{\circ} \mathrm{C}$ ) and a thermostatted hood were used. The sample $\left(c=1 \mathrm{mg} \mathrm{ml}{ }^{-1}\right)$ was left to equilibrate for 30 minutes at $2{ }^{\circ} \mathrm{C}$ before the start of the heating and cooling cycle with a heating rate of $20{ }^{\circ} \mathrm{C} \mathrm{h}^{-1}$. To prevent the evaporation of water, the cone and gap were covered with small amounts of low viscosity silicone oil.

\section{Results and discussion}

\subsection{Aggregation behaviour of DMAPPC-C32-POH in aqueous suspension}

The asymmetrical bolaamphiphile DMAPPC-C32-POH (Fig. 1) contains two headgroups, which have net charges depending on the $\mathrm{pH}$ value of the aqueous suspension. The exact $\mathrm{p} K_{\mathrm{a}}$ values of these groups could not be determined, but it is possible to deduce the probable protonation behaviour from values of similar molecules.

The two phosphate groups of DMAPPC-C32-POH can be expected to be deprotonated and therefore negatively charged at $\mathrm{pH}$ values between 4 and 12 . This is due to the low $\mathrm{p} K_{\mathrm{a}}$ value of this group, which has been determined to be 3.3 for the suspended bolaamphiphile $\mathrm{Me}_{2} \mathrm{PE}-\mathrm{C} 32-\mathrm{Me}_{2} \mathrm{PE}$ containing phosphodiester groups in similar chemical surroundings. ${ }^{3}$

The dimethylamino group at the end of the bolaamphiphile, however, changes its protonation state in the $\mathrm{pH}$ range from 5 to 10. For the dimethylamino group of $\mathrm{Me}_{2} \mathrm{PE}-\mathrm{C} 32-\mathrm{Me}_{2} \mathrm{PE}$ a titration experiment in $150 \mathrm{mM} \mathrm{KCl}$ solution showed that it has an apparent $\mathrm{p} K \mathrm{a}_{\mathrm{a}}^{\text {app }}$ value of $6.5 .^{3}$ Without additional salt in the suspension, i.e. at low ionic strength, the $\mathrm{p} K_{\mathrm{a}}^{\text {app }}$ values tend to increase to $10-11$ as determined by DSC measurements. ${ }^{23}$ This shift in $\mathrm{p} K_{\mathrm{a}}^{\mathrm{app}}$ is caused by the accumulation of charged headgroups at the fibre surface. The presence of buffer salts screens these charges and $\mathrm{p} K_{\mathrm{a}}^{\mathrm{app}}$ is shifted to lower values with increasing salt concentration.

In $\mathrm{Me}_{2} \mathrm{PE}-\mathrm{C} 32-\mathrm{Me}_{2} \mathrm{PE}$ the dimethylamino group is in close proximity to the negatively charged phosphodiester group whereas in the DMAPPC headgroup the dimethylamino group is located next to the positively charged quaternary ammonium group. This might influence the intrinsic $\mathrm{p} K_{\mathrm{a}}$ value, making a direct comparison between the two compounds difficult. It is likely that the intrinsic $\mathrm{p} K_{\mathrm{a}}$ value of the DMAPPC headgroup is somewhat lower, as protonation of the dimethylamino group next to the positive charge is more difficult.

The possible protonation states at two different $\mathrm{pH}$-values are shown in Table 1. For the experiments the bolaamphiphiles were suspended in acetate buffer at pH $5\left(\mathrm{H}_{2} \mathrm{O}\right.$ or $\left.\mathrm{D}_{2} \mathrm{O}\right)$ and in carbonate buffer at $\mathrm{pH} 10\left(\mathrm{H}_{2} \mathrm{O}\right)$, respectively, to investigate the influence of the protonation state on the selfassembly process. $\mathrm{D}_{2} \mathrm{O}$ was utilized for the neutron scattering experiments and the connection between the $\mathrm{pD}$ value and the $\mathrm{pH}$ value is $\mathrm{pD}=\mathrm{pH}+0.4 .^{24}$

3.1.1 TEM. When suspended in water, the bolaamphiphile DMAPPC-C32-POH formed gels at room temperature at low $\mathrm{pH}$ value. This indicates that self-assembly leads again to a network of nanofibres as observed before for similar bolaamphiphiles. Transmission electron microscopy (TEM) was used to visualize the aggregate structures in the aqueous suspensions. TEM images of samples prepared at room temperature are shown in Fig. 2.

Table 1 Schematic representation of the protonation states of DMAPPC-C32$\mathrm{POH}$ and DMAPPC-C32-OH at different $\mathrm{pH}$ values

\begin{tabular}{|c|c|c|c|}
\hline Bolaamphiphile & $\begin{array}{l}\mathrm{pH} \\
\text { value }\end{array}$ & $\begin{array}{l}\text { Sketch of } \\
\text { bolaamphiphile }\end{array}$ & $\begin{array}{l}\text { Overall net } \\
\text { charge }\end{array}$ \\
\hline \multirow[t]{2}{*}{ DMAPPC-C32-POH } & 5 & $+-\odot \quad-$ & \pm 0 \\
\hline & 10 & $0+-$ & -1 \\
\hline \multirow[t]{2}{*}{ DMAPPC-C32-OH } & 5 & ++- & +1 \\
\hline & 10 & $0+-$ & \pm 0 \\
\hline
\end{tabular}



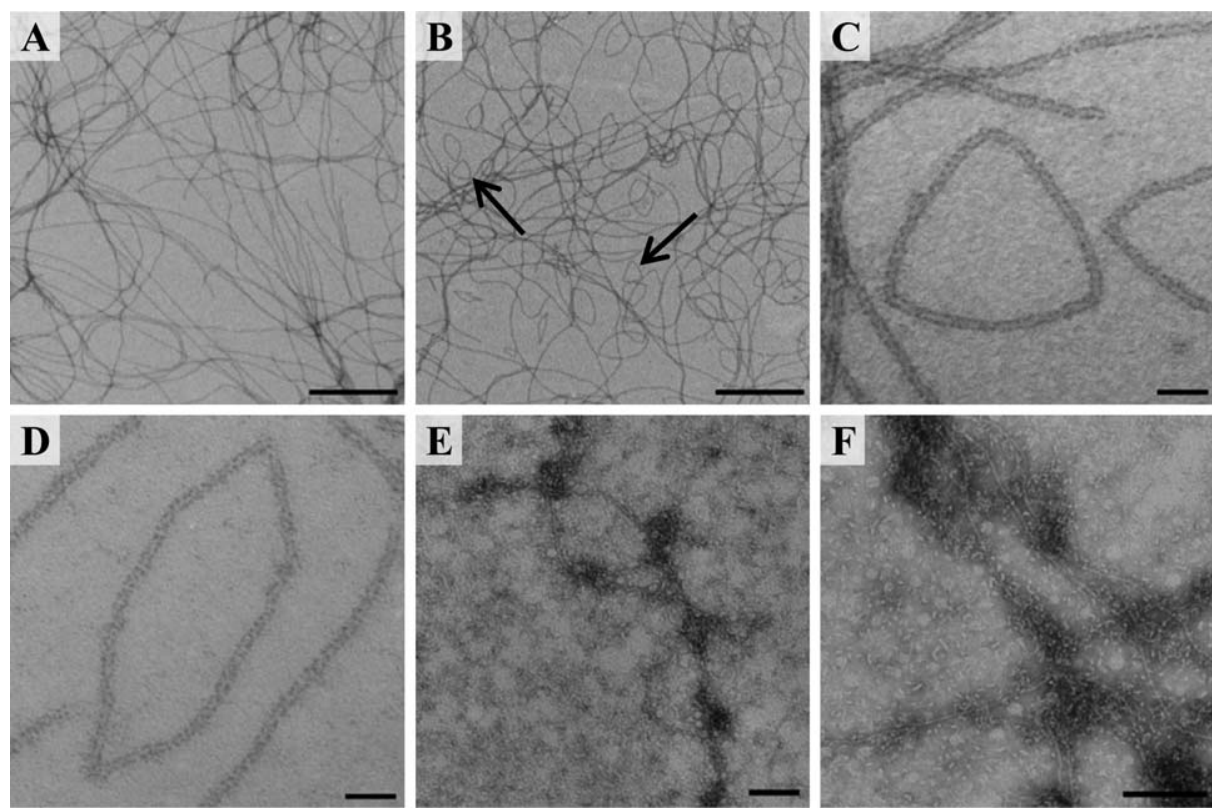

Fig. 2 TEM images of aqueous DMAPPC-C32-POH suspensions in (A) water, (B-D) acetate buffer at $\mathrm{pH} 5$ and (E and F) carbonate buffer at pH 10 prepared at $25^{\circ} \mathrm{C}$. The samples were stained with uranyl acetate. The bar corresponds to $500 \mathrm{~nm}$ ( $A$ and B), to $25 \mathrm{~nm}$ (C and D) and to $100 \mathrm{~nm}$ (E and F), respectively.

The TEM image of the suspension in water shows the presence of long, regular fibres with a diameter of approximately 6 $\mathrm{nm}$ (Fig. 2A). TEM images of the suspension in acetate buffer at pH 5 show next to long fibres with a diameter of approximately 6 $\mathrm{nm}$ many fibres that have formed irregular, circular structures (black arrows in Fig. 2B). At higher magnification (see Fig. 2C and D), it seems as if they are composed of short fibre segments that overlap and are probably connected at the ends. This was observed in several independently prepared samples, thus preparation artefacts can be ruled out. These structures have not been observed in any other bolaamphiphile suspensions so far and seem to be a specific property of the asymmetrical molecule DMAPPC-C32-POH in acetate buffer at $\mathrm{pH}$ 5. At $\mathrm{pH} 10$, the bola molecules carry an overall negative charge resulting in a repulsive interaction between headgroups. The stability of the fibrous aggregates is therefore strongly decreased. The TEM images show the presence of mainly short fibre segments or elongated/ellipsoidal micelles (see Fig. 2E and F).

3.1.2 DSC and FT-IR. To investigate the aggregation behaviour of DMAPPC-C32-POH in aqueous suspension as a function of temperature, DSC and FT-IR measurements were carried out with suspensions in acetate buffer at $\mathrm{pH} 5$ and in carbonate buffer at $\mathrm{pH}$ 10. The results are shown in Fig. 3.

The DSC curve of the suspension in acetate buffer at $\mathrm{pH} 5$ shows three endothermic peaks at 45.1, 65.5, and $84.2{ }^{\circ} \mathrm{C}$, respectively, with transition enthalpies $\Delta H$ of $\sim 13, \sim 10$, and $\sim 6.5 \mathrm{~kJ} \mathrm{~mol}^{-1}$, i.e., with a total transition enthalpy of $c a .30 \mathrm{~kJ}$ $\mathrm{mol}^{-1}$ (Fig. 3A). The corresponding cooling curve shows the same peaks with a small hysteresis of the second one. The thermal behaviour observed for DMAPPC-C32-POH suspensions is very similar to the one observed for the symmetrical bolaamphiphile $\mathrm{Me}_{2} \mathrm{PE}-\mathrm{C} 32-\mathrm{Me}_{2} \mathrm{PE}$ suspended in acetate buffer
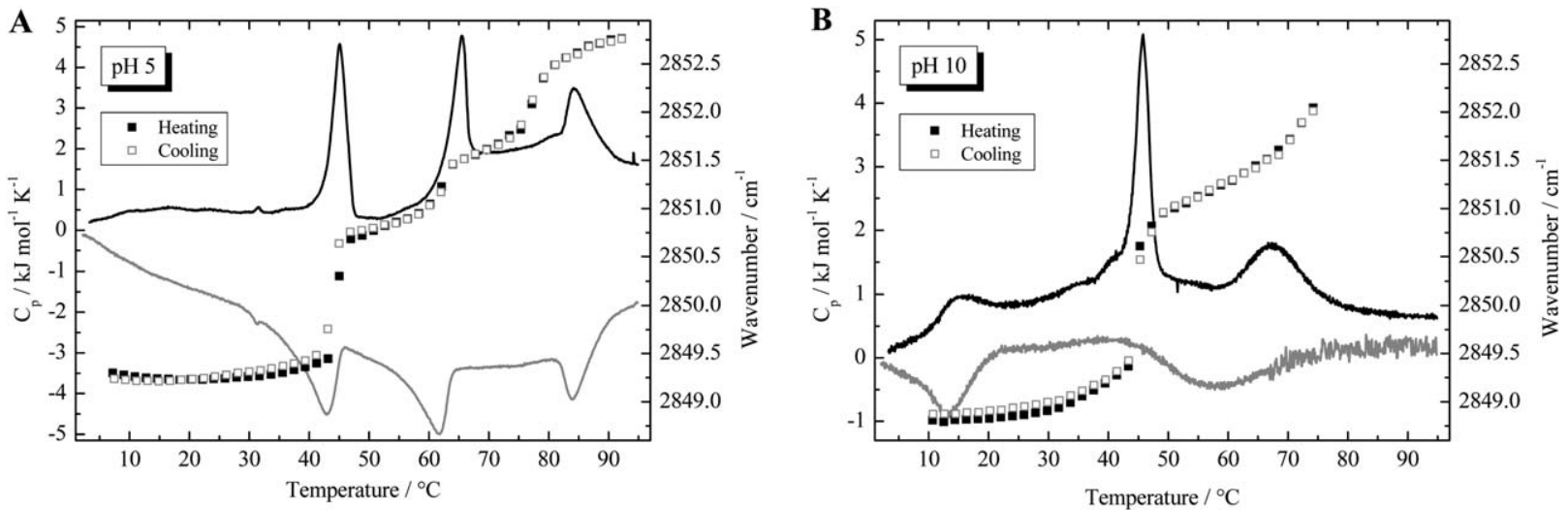

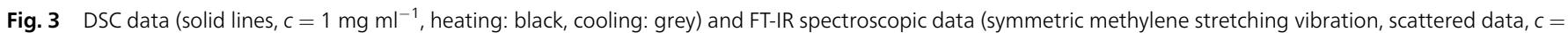
$50 \mathrm{mg} \mathrm{ml}^{-1}$; heating: filled squares, cooling: open squares) of DMAPPC-C32-POH in (A) acetate buffer at pH 5 and (B) carbonate buffer at pH 10. 
at $\mathrm{pH} 5 .^{3}$ For this bolaamphiphile also three thermal transitions with very similar transition temperatures were observed. However, the total transition enthalpy of about $43 \mathrm{~kJ} \mathrm{~mol}^{-1}$ was almost $40 \%$ higher than for DMAPPC-C32-POH at $\mathrm{pH}$. For $\mathrm{Me}_{2} \mathrm{PE}-\mathrm{C} 32-\mathrm{Me}_{2} \mathrm{PE}$ the first thermal transition corresponds to a fibre-fibre transition, the second one to a fibre-micelle and the third to a transition between two different types of micelles. ${ }^{3,22}$

The FT-IR measurements reveal wavenumbers below 2850 $\mathrm{cm}^{-1}$ for the symmetric methylene stretching vibrational band up to $45^{\circ} \mathrm{C}$. This is an indication of an all-trans conformation in the alkyl chain needed for the formation of the previously described fibre structure.,12,13,22 The three step behaviour of the wavenumber shows an increase in the amount of gauche conformers, which is in accordance with the interpretation of the three transitions as a fibre-fibre transition followed by a fibre-micelle transition and the transition between two different types of micelles., ${ }^{3,12,13,22}$ It is therefore likely that DMAPPC-C32-POH shows the same sequence of types of aggregates as observed before for $\mathrm{Me}_{2} \mathrm{PE}-\mathrm{C} 32-\mathrm{Me}_{2} \mathrm{PE}$.

A different thermal behaviour is found when DMAPPC-C32$\mathrm{POH}$ is suspended in carbonate buffer at $\mathrm{pH} 10$. At this $\mathrm{pH}$ value the dimethylamino moieties of the DMAPPC headgroup are deprotonated leading to a zwitterionic DMAPPC headgroup and an overall negatively charged bola molecule (see Table 1). The

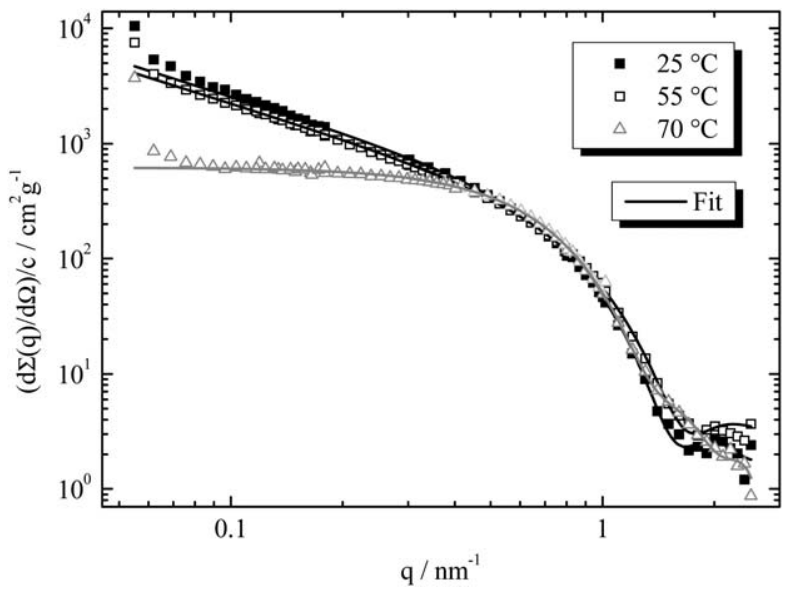

Fig. 4 SANS data of suspensions of DMAPPC-C32-POH in acetate buffer at $\mathrm{pH} 5$ measured at different temperatures with IFT fits (solid lines).
DSC heating curve showed three endothermic transitions at 15, 45.8 , and about $67^{\circ} \mathrm{C}$, respectively (Fig. 3B). This decrease in the transition temperatures can be explained by repulsive interactions between the bola molecules due to the negatively charged phosphoric acid diester group. The FT-IR data for the wavenumber of the symmetric methylene stretching vibration show only a very slight increase of the frequency at the first DSC peak; the major change occurs at the second peak connected with the fibre to micelle transition. The third transition from micelle I to micelle II is also visible. The overall change in wavenumber is similar to the one found for the sample at pH 5. Similar results were obtained for suspension of the symmetrical bolaamphiphile $\mathrm{Me}_{2} \mathrm{PE}-\mathrm{C} 32-\mathrm{Me}_{2} \mathrm{PE}$ in buffer at $\mathrm{pH} 10$ where the bolalipid also carries a negative charge in the headgroup. ${ }^{3}$

3.1.3 SANS. To get further insight into the structure of the fibres, SANS measurements were performed at three temperatures to investigate the changes of the aggregate structures. In acetate buffer with $\mathrm{D}_{2} \mathrm{O}$ as the solvent, the $\mathrm{pH}$ value was adjusted to 5 . The scattering data of the SANS experiments at different temperatures and their fits are displayed in Fig. 4.

The fits were performed using the IFT method described in detail before ${ }^{\mathbf{1 2 , 2 0 - 2 2}}$ and are in good agreement with the experimental data. For fitting the data of the measurements at 25 and $55{ }^{\circ} \mathrm{C}$, the model of infinitely long cylinders was used and for the data at $70^{\circ} \mathrm{C}$ spherical aggregates were assumed. The results of the fits are provided in Table 2.

The results of the fits support the interpretation of the aggregates structure as fibres below the first and the second DSC transition peak. The radius of cross-section of the fibres is in accordance with the one determined from the TEM images (Fig. 2). At $55{ }^{\circ} \mathrm{C}$ the radius becomes smaller due to the higher amount of gauche conformers in the alkyl spacer chain reducing the effective length of the spacer chain. The values determined for a suspension of the symmetrical bolaamphiphile $\mathrm{Me}_{2} \mathrm{PE}$ C32- $\mathrm{Me}_{2} \mathrm{PE}$ in acetate buffer at pH 5 (ref. 22) are provided in Table 2 for comparison. The size of the aggregates is very similar to the one determined for the DMAPPC-C32-POH suspensions. Modelling of scattering curves by stiff cylinders with elliptical cross-section and ellipsoids of revolution confirms that the cross-section is slightly elliptical with an axial ratio less than 1.4 for DMAPPC-C32-POH in acetate buffer ( $\mathrm{pH} 5)$ at $25^{\circ} \mathrm{C}$ (see the $\mathrm{ESI} \dagger$ ). The value of the smaller semi-axis (about $2.1 \mathrm{~nm}$ ) practically does not change during transformation from fibres to

Table 2 Data obtained from IFT fits of suspensions of DMAPPC-C32-POH in acetate buffer at $\mathrm{pH} 5^{\mathrm{a}}$

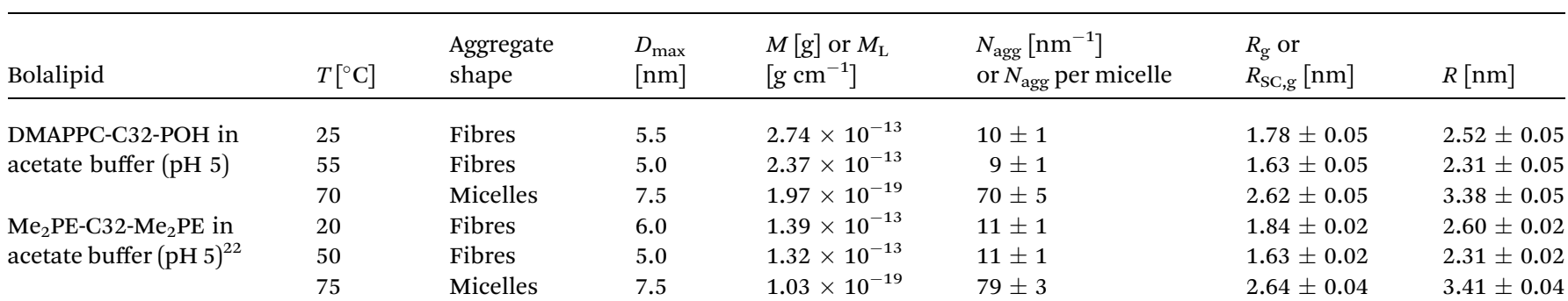

${ }^{a} D_{\text {max }}:$ maximal size or cross-section of aggregate, $M$ : mass, $M_{\mathrm{L}}$ : mass per unit length, $N_{\text {agg: }}$ aggregation number, $R_{\mathrm{g}}$ : radius of gyration, $R_{\mathrm{SC}, \mathrm{g}}:$ radius of gyration of cross-section, $R$ : radius. 
A

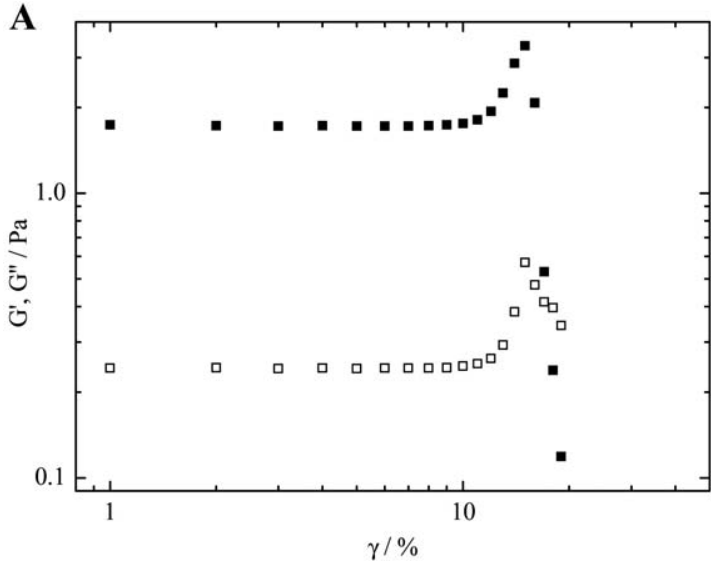

B

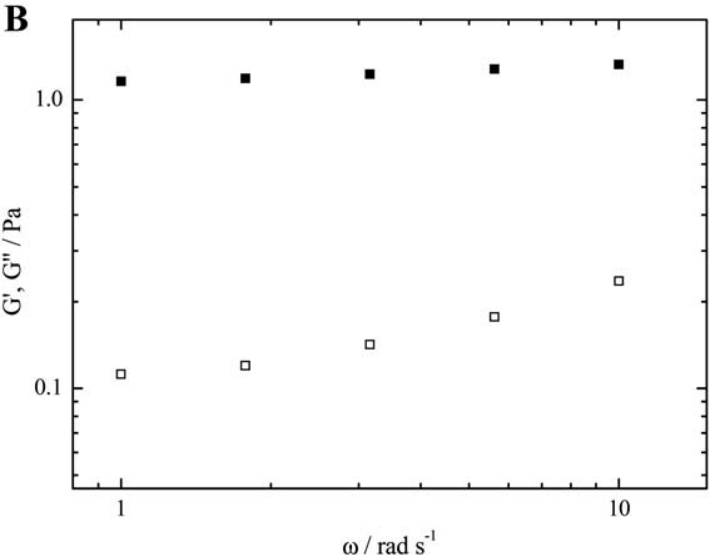

Fig. 5 (A) Amplitude sweeps of DMAPPC-C32-POH suspensions ( $c=1 \mathrm{mg} \mathrm{ml}^{-1}$ ) in acetate buffer at $\mathrm{pH} 5$ at $20{ }^{\circ} \mathrm{C}\left(\omega=10 \mathrm{rad} \mathrm{s}^{-1}\right)$. (B) Frequency sweeps of DMAPPC-C32-POH suspensions in acetate buffer at pH 5 at $20^{\circ} \mathrm{C}(\gamma=1 \%) . G^{\prime}$ : filled symbols, $G^{\prime \prime}$ : open symbols.

ellipsoid like aggregates with increasing temperature (see Table $\mathrm{S} 1 \dagger$ ). For DMAPPC-C32-POH the length of the shorter axis (a) of the elliptical fibre is higher than the value found for $\mathrm{Me}_{2} \mathrm{PE}-\mathrm{C} 32$ $\mathrm{Me}_{2} \mathrm{PE}$ fibres; the mass fraction of bola molecules, however, is slightly lower for DMAPPC-C32-POH. This could be due to a different tilt of the molecules in the fibres relative to the fibre axis (see Table $\mathrm{S} 1 \dagger$ ).

3.1.4 Rheology. Oscillatory rheological measurements were used to examine the properties of the hydrogels formed by
DMAPPC-C32-POH in buffer at $\mathrm{pH}$ 5. The results of the amplitude and frequency sweeps for the determination of the linear viscoelastic (LVE) region are provided in Fig. 5. The amplitude sweep shows that the storage modulus $G^{\prime}$ is larger than the loss modulus $G^{\prime \prime}$ at low deformations. This is the typical behaviour ascribed to viscoelastic gels. ${ }^{25,26}$

An important property of the DMAPPC-C32-POH suspensions is the straining limit indicated by the crossover from gel $\left(G^{\prime}>G^{\prime \prime}\right)$ to sol $\left(G^{\prime}<G^{\prime \prime}\right)$. For the suspension in acetate buffer at $\mathrm{pH} 5$, this limit is found at $20 \%$ deformation. Strain stiffening can also be observed in the suspension as can be seen in the increase in $G^{\prime}$ and $G^{\prime \prime}$ just before the crossover point. This phenomenon has also been observed in samples of symmetrical bolaamphiphiles ${ }^{23}$ as well as in biological systems of collagen or actin fibres. ${ }^{27}$

The frequency sweep was carried out with a deformation of $1 \%$. In the frequency range from 1 to $10 \mathrm{rad} \mathrm{s}^{-1}$ the values of $G^{\prime}$ remain constant and the values of $G^{\prime \prime}$ show a slight increase. $G^{\prime}$ is larger than $G^{\prime \prime}$ over the whole range indicating the presence of a viscoelastic gel. The LVE region reaches up to $10 \%$ deformation and up to an angular frequency of $10 \mathrm{rad} \mathrm{s}^{-1}$.

The suspension shows a high gel strength and elasticity for the sample at $\mathrm{pH} 5$ inside the LVE region. However, the low straining limit indicates a certain inflexibility of the fibres to deformation or easy breaking of the cross-links and entanglements under high straining conditions. This is a common behaviour also of crystalline nanofibres such as 12-hydroxystearic acid (HSA), which can have straining limits as low as $2 \%$ and break easily. ${ }^{28}$ The TEM images of the suspension of DMAPPC$\mathrm{C} 32-\mathrm{POH}$ at $\mathrm{pH} 5$ support this observation as short fibre segments are present and entanglements between these segments can be supposed to break more easily under strain than in suspensions with longer fibres (see Fig. 2B-D).

For the suspension at $\mathrm{pH} 10$ no rheological measurements were performed, because the suspensions did not show significant gelation behaviour. Based on the TEM images it is clear that a large amount of small aggregates is present in the suspension so that gelation by entanglement of fibres becomes impossible.

Temperature dependent rheological measurements inside the LVE region were performed with a suspension of DMAPPC-C32$\mathrm{POH}$ in acetate buffer at $\mathrm{pH}$. The change in $G^{\prime}$ and $G^{\prime \prime}$ was monitored in the temperature range between 20 and $75^{\circ} \mathrm{C}$ and is shown in Fig. S1 (ESI $\dagger$ ). The values of $G^{\prime}$ and $G^{\prime \prime}$ decrease in two
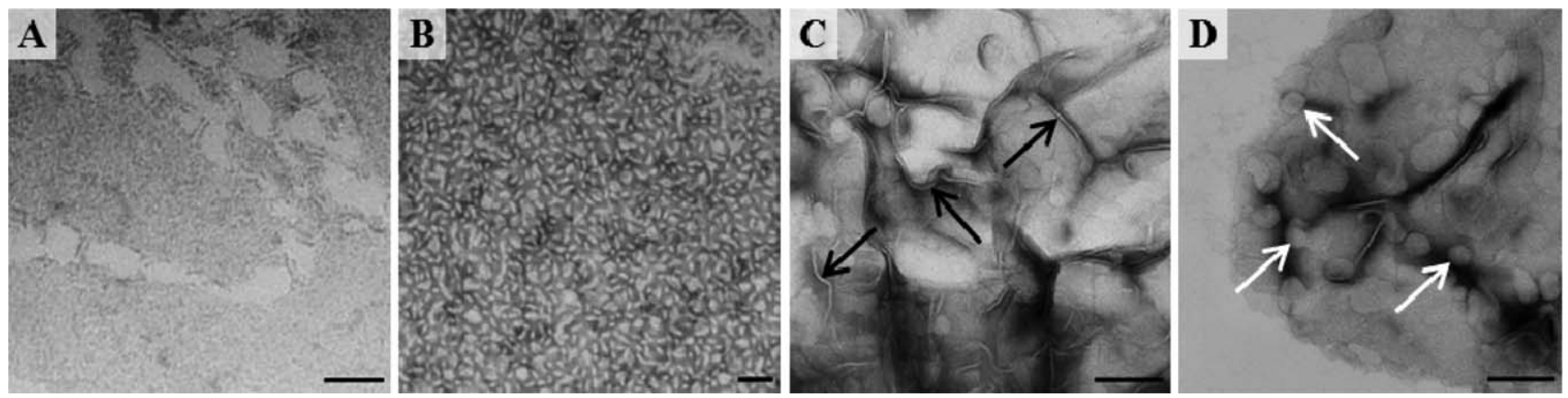

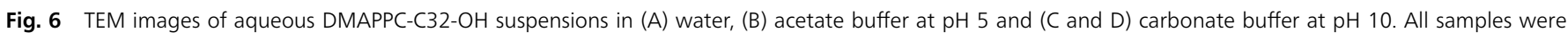
prepared at $25^{\circ} \mathrm{C}$ and were stained with uranyl acetate. The bar corresponds to $50 \mathrm{~nm}(\mathrm{~A}$ and $\mathrm{B}$ ) and to $100 \mathrm{~nm}(\mathrm{C}$ and $\mathrm{D}$ ). 


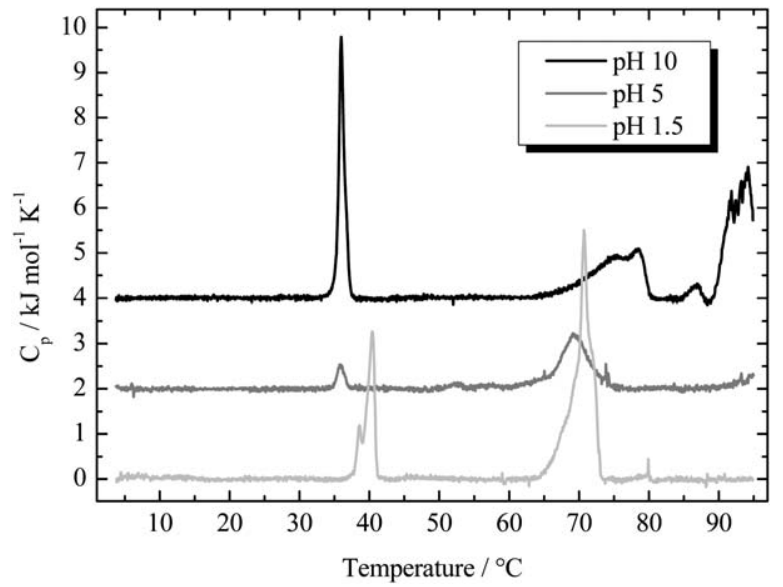

Fig. 7 DSC heating scans of DMAPPC-C32-OH suspensions $\left(c=1 \mathrm{mg} \mathrm{ml}^{-1}\right)$ in carbonate buffer at $\mathrm{pH} 10$ (black), acetate buffer at $\mathrm{pH} 5$ (grey) and in water at pH 1.5 (light grey). The heating rate was $20^{\circ} \mathrm{Ch}^{-1}$. The curves are vertically shifted for clarity.

steps around 40 and $60{ }^{\circ} \mathrm{C}$, which is in accordance with the transition peaks observed in the DSC measurements (see Fig. 3A). $G^{\prime}$ is about one order of magnitude higher than $G^{\prime \prime}$ up to $60{ }^{\circ} \mathrm{C}$. Above this temperature both moduli have about the same value. This proves the presence of a viscoelastic gel state up to $60^{\circ} \mathrm{C}$ and supports the interpretation of the DSC peaks as a fibre-fibre transition followed by a fibre-micelle transition. At higher temperature the suspension has the properties of a Newtonian fluid. ${ }^{29}$

Compared to the gel of $\mathrm{Me}_{2} \mathrm{PE}-\mathrm{C} 32-\mathrm{Me}_{2} \mathrm{PE}$ in acetate buffer at $\mathrm{pH} 5$ where additional stabilization of the fibre network can occur via hydrogen bonds, ${ }^{3}$ the gel of DMAPPC-C32-POH is weak, but it is a little stronger than the gel formed by $\mathrm{Me}_{2} \mathrm{PE}$ C32- $\mathrm{Me}_{2} \mathrm{PE}$ at $\mathrm{pH} 11$ in the presence of divalent ions shielding the negatively charged headgroups. ${ }^{23}$ The second plateau value of $G^{\prime}$ has about the same value as observed in the case of $\mathrm{Me}_{2} \mathrm{PE}$ $\mathrm{C} 32-\mathrm{Me}_{2} \mathrm{PE}$ at $\mathrm{pH} 11$ with divalent ions and seems to be a state of intermediate gel stability formed at higher temperature or insufficient shielding of opposing charges. ${ }^{23}$

\subsection{Aggregation behaviour of DMAPPC-C32-OH in aqueous suspension}

As a second asymmetric bolaamphiphile we investigated the compound DMAPPC-C32-OH, having a much higher asymmetry in headgroup size, as at one end the polar group consists only of a hydroxyl group (see Fig. 1). As the $\mathrm{OH}$ headgroup carries no charge, DMAPPC-C32-OH at pH 5 is therefore positively charged and uncharged at pH 10 (see Table 1).

3.2.1 TEM. When the bolaamphiphile DMAPPC-C32-OH was suspended in water it formed no gels at room temperature both at low as well as high $\mathrm{pH}$ values. The TEM images in Fig. 6A prepared from DMAPPC-C32-OH in water at room temperature show a mixture of lamellar aggregates together with shorter undefined aggregates (approx. $25 \mathrm{~nm}$ ). When acetate buffer at pH 5 was used (Fig. 6B) also a mixture of lamellar aggregates and short elongated micellar type aggregates (approx. $25 \mathrm{~nm}$ ) is seen. In contrast, the images of samples prepared in carbonate buffer at pH 10 (Fig. 6C and D) clearly exhibit the presence of larger lamellar sheets or vesicles that are sometimes folded (see black arrows in Fig. 6C) due to the drying procedure during the sample preparation. Besides the large sheet-like structures, some smaller and nearly rounded lamellar aggregates are visible (see white arrows in Fig. 6D). The TEM images therefore show that for this highly asymmetric bolaamphiphile fibre formation does not occur. The aggregation process leads to lamellar aggregates instead.

3.2.2 DSC and FT-IR. We used DSC and FT-IR to study the thermotropic behaviour of these aggregates. The DSC heating scans of DMAPPC-C32-OH suspended in acetate buffer at $\mathrm{pH} 5$, carbonate buffer at $\mathrm{pH} 10$, and in water at $\mathrm{pH} 1.5$ are displayed in Fig. 7. In the DSC heating curve of the suspension at pH 5 two peaks appear at 35.9 and $69.3{ }^{\circ} \mathrm{C}$ (Fig. 7, grey curve). To test whether the charge of the headgroup has an effect on the thermotropic behaviour we performed additional DSC experiments at $\mathrm{pH} 10$ and $\mathrm{pH}$ 1.5. For the suspension in carbonate buffer at pH 10 (Fig. 7, black curve) one large endothermic peak at $36.0{ }^{\circ} \mathrm{C}$, a second one at $78.5{ }^{\circ} \mathrm{C}$ (with a small shoulder at lower temperatures), a small peak at $86.9^{\circ} \mathrm{C}$, and the onset of another peak (at $91.8{ }^{\circ} \mathrm{C}$ ) are observed, whereas for the sample
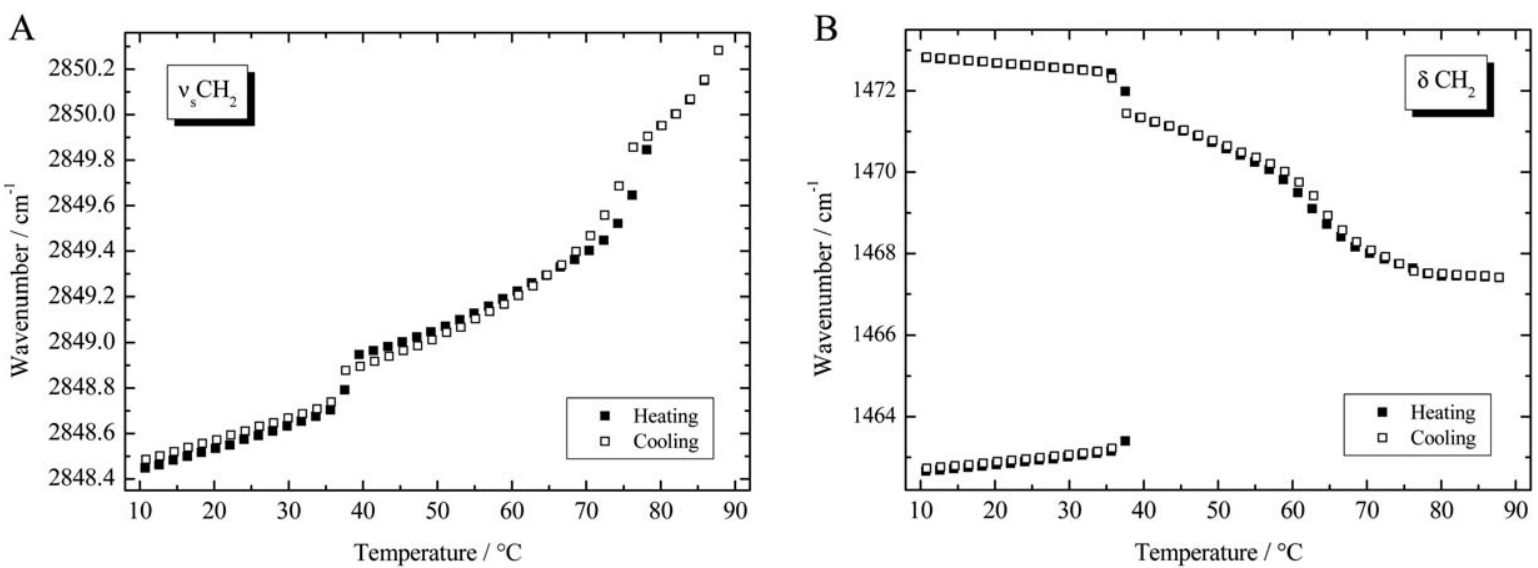

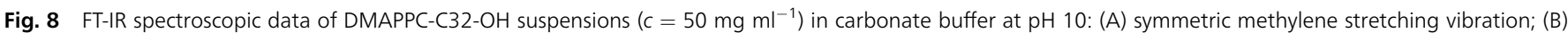
methylene scissoring vibration. Heating: filled squares, cooling: open squares. 


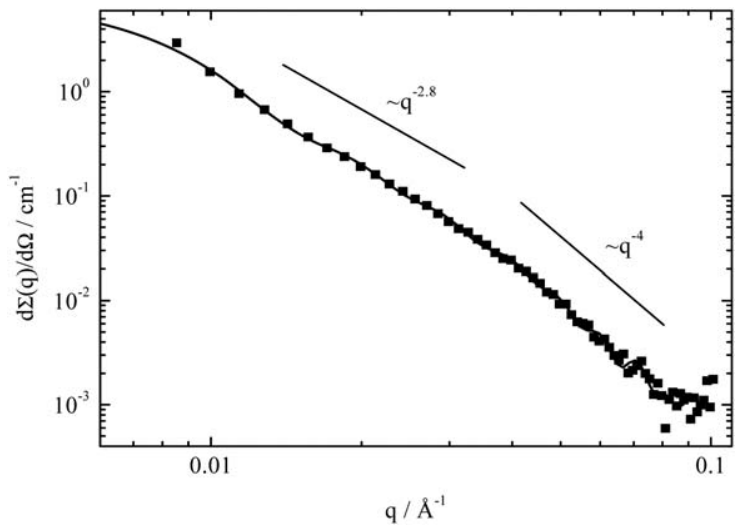

Fig. 9 SAXS profile of an aqueous DMAPPC-C32-OH suspension ( $c=10 \mathrm{mg}$ $\mathrm{ml}^{-1}$ ) measured at $25^{\circ} \mathrm{C}$. The data (lower $q$ part up to $0.08 \AA^{-1}$ ) were analysed by the IFT method (solid line).

at pH 1.5 (Fig. 7, light grey curve), where the DMAPPC headgroup is definitely positively charged, the sequence of transitions is similar to the one for the sample at $\mathrm{pH} 5$, only that the transition temperatures are slightly increased and the transition enthalpies are higher.

To identify the nature of these thermotropic transitions we performed FT-IR-experiments. For the suspension of DMAPPC$\mathrm{C} 32-\mathrm{OH}$ in carbonate buffer at $\mathrm{pH} 10$, the wavenumber of the symmetric methylene stretching vibration as a function of temperature is shown in Fig. 8A together with the wavenumber of the methylene scissoring vibration (Fig. 8B) (see also spectra in Fig. S2 $\dagger$ ). The results clearly show a stepwise change in the frequency of the symmetric $\mathrm{CH}_{2}$-stretching band at those temperatures where thermotropic transitions were observed by DSC, namely $35{ }^{\circ} \mathrm{C}$ and $76{ }^{\circ} \mathrm{C}$. However, the wavenumber stays at low values characteristic of an all-trans alkyl chain indicating that no "melting" of the alkyl chains occurs at these temperatures. The $\mathrm{CH}_{2}$-deformation band shows a characteristic splitting at low temperature indicating an orthorhombic packing of the alkyl chain. When the temperature is increased, this splitting disappears at a temperature coinciding with the first thermotropic transition. These results clearly show that the first two transitions at lower temperature are due to different chain packing modes in ordered lamellar phases. At the low temperature transition the orthorhombic packing of the chains is lost and a hexagonal packing of the chains appears. At the second transition, the chains become slightly more disordered but are still not liquid-crystalline. The third, high temperature transition (not fully accessible by FT-IR and DSC experiments) is probably a transition to a liquid-crystalline state. The DSC and FT-IR results together with the TEM images suggest that only lamellar phases are formed by this bolaamphiphile, probably by interdigitation of the alkyl chains, and that in the low temperature lamellar phase the chains are well ordered in an orthorhombic packing. This gets lost upon heating at a well-defined temperature, namely ca. $35{ }^{\circ} \mathrm{C}$ for the sample at $\mathrm{pH} 10$ and $37{ }^{\circ} \mathrm{C}$ for the sample at $\mathrm{pH} 5$ (see Fig. S3†). Therefore, at high $\mathrm{pH}$, where the DMAPPC headgroup is zwitterionic and the bola molecule carries no net charge, large lamellar structures are the predominant aggregate form.

3.2.3 SAXS. To get further information about the structure of the aggregates formed from DMAPPC-C32-OH in aqueous suspension X-ray scattering measurements were performed. Fig. 9 shows that the scattering is due to the formation of large aggregates. However, the scattering from whole objects (Guinier approximation, eqn (1)) cannot be observed due to limited $q_{\min }$.

$$
\frac{\mathrm{d} \sum(q)}{\mathrm{d} \Omega}=\frac{\mathrm{d} \sum(0)}{\mathrm{d} \Omega} \mathrm{e}^{-\frac{q^{2} R_{\mathrm{g}}{ }^{2}}{3}}
$$

The objects are too large for the investigated $q$ interval; instead, the scattering from parts of the objects or object interfaces is observed. In the high $q$ region one can observe a scattering intensity proportional to $q^{-4}$ and this is attributed to the scattering of the interface between aggregates and solvent. At lower $q$ values a scattering intensity proportional to $q^{-2.8}$ was observed, somehow intermediate between the scattering intensity of whole objects and the interface. The scattering curve was analyzed using the IFT method and an estimation of the lower limit of the size of these aggregates gave a value of $60 \mathrm{~nm}$.
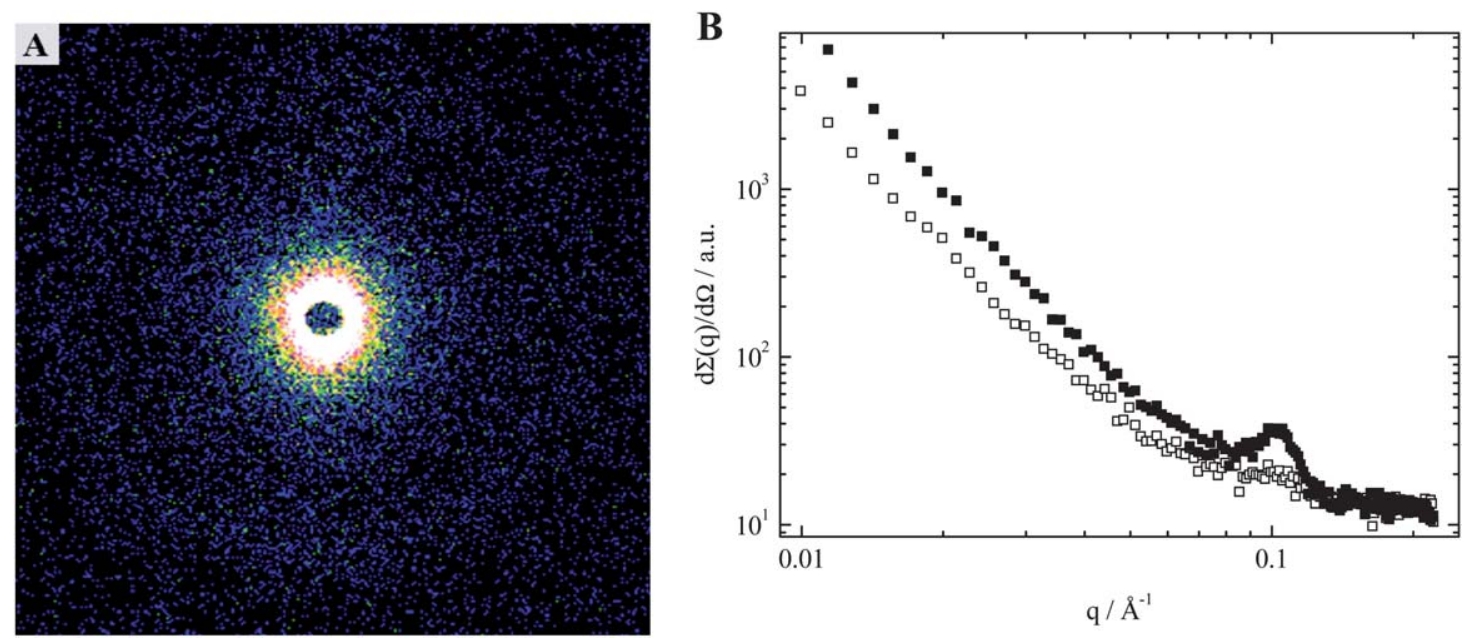

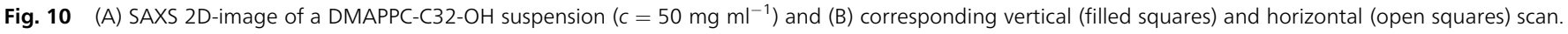




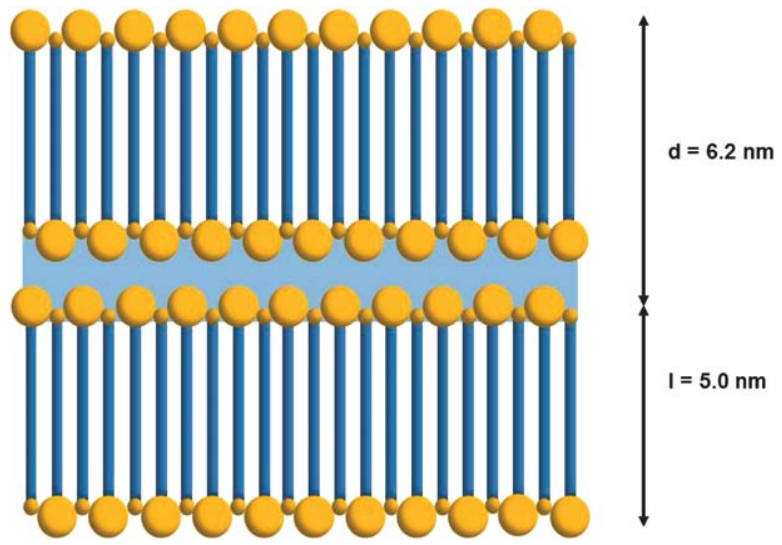

Fig. 11 Scheme of a lamellar phase consisting of interdigitated bolaamphiphile molecules in a monolayer arrangement.

The TEM images (see Fig. 6) showed that the objects formed by DMAPPC-C32-OH in aqueous suspension ( $\mathrm{pH} 10)$ are lamellar-like aggregates. Additional SAXS measurements were therefore performed with a sample with higher concentration of DMAPPC-C32-OH. The results supported the previous assumptions. Asymmetrical 2D-images were observed in this case and an orientation of lamellar layers seemed to occur (see Fig. 10). At high $q$ values, the interference maximum at $q_{\max }=$ $0.1 \AA^{-1}$ (observed only in one direction) points to stacked lamellar-like structures and corresponds to the repeat distance $d$ between lamellar sheets of $d=2 \pi / q_{\max }=6.2 \pm 0.1 \mathrm{~nm}$. As the length of the molecule is about $5 \mathrm{~nm}$ this repeat distance of $6.2 \mathrm{~nm}$ supports a packing model of a monolayer with alternating headgroups and alternating interdigitated chains with a perpendicular orientation relative to the monolayer plane. A water layer thickness of $1.2 \mathrm{~nm}$ would probably be sufficient for a complete hydration of the headgroup region (see Fig. 11).

A similar symmetrical arrangement was found for much shorter single chain bolaamphiphiles with a chain length up to 16 methylene groups by Masuda and Shimizu, ${ }^{15,16}$ which formed microtubes consisting of monolayer membranes. In the crystal, however, the molecules were found to be tilted relative to the monolayer normal, leading to a reduction of the periodicity $d$. For the related compound $\omega$-hydroxybehenylphosphocholine (PC-C22-OH), which is more related to our molecule DMAPPCC32-OH, Pohle et al. ${ }^{30}$ suggested also an interdigitated monolayer structure, however, only based on the analysis of Raman and IR spectra. Other asymmetric bolaamphiphiles, such as those derived from archaebacteria, can also form interdigitated monolayer membranes. ${ }^{31}$

\section{Conclusions}

Asymmetrical bolaamphiphiles can form fibre-like structures or lamellar phases depending on the size difference between the two headgroups of the bolaamphiphile. For DMAPPC-C32-POH in acetate buffer at pH 5 and in carbonate buffer at pH 10 we observed fibres as the predominant aggregate form at low temperature. The fibres contain bolaamphiphiles with alkyl chains in the all-trans conformation and they exhibit a fibrefibre transition as also described for the symmetrical molecule $\mathrm{Me}_{2} \mathrm{PE}-\mathrm{C} 32-\mathrm{Me}_{2} \mathrm{PE}^{3}$ SANS measurements showed that the fibres are comparable in their aggregation numbers to fibres formed by $\mathrm{Me}_{2} \mathrm{PE}-\mathrm{C} 32-\mathrm{Me}_{2} \mathrm{PE}$ at $\mathrm{pH}$ 5. In the suspensions at $\mathrm{pH}$, the fibres of DMAPPC-C32-POH showed an increased tendency to break into smaller segments that formed irregular, circular aggregates.

Rheological measurements revealed similar viscoelastic gel behaviour for the DMAPPC-C32-POH fibres compared to $\mathrm{Me}_{2} \mathrm{PE}$ $\mathrm{C} 32-\mathrm{Me}_{2} \mathrm{PE}$ fibres at $\mathrm{pH} 5$ but with lower strain resistance due to the presence of the short fibre segments that decrease the stability of the entanglements supporting the formation of viscoelastic gels. At pH 10 only short fibre segments are formed due to the fact that the overall charge of the molecule is now -1 . As a consequence no gel formation is observed and the suspension behaves as a Newtonian fluid.

In the asymmetrical bolaamphiphile DMAPPC-C32-OH the headgroups have a larger size difference than in the molecule DMAPPC-C32-POH. The formation of lamellar structures of different sizes is observed in DMAPPC-C32-OH suspensions at high $\mathrm{pH}$-values whereas at lower $\mathrm{pH}$-values, where the molecules are positively charged, some other aggregate structures besides lamellar aggregates are seen in TEM images. In the lamellar aggregates or phases the thickness of the lamellae indicates a monolayer of molecules with interdigitated alkyl chains and orientation of the chains perpendicular to the lamellae. The FT-IR results support this assumption, as the appearance of a splitting of the methylene scissoring bands at low temperature indicates an orthorhombic packing of the chains with high order. However, this high order is lost upon heating to the first thermotropic transition where a transformation into a hexagonal chain packing is observed. Due to the long $\mathrm{C}_{32}$ chains, the melting into liquid-crystalline phases occurs at very high temperature above $90{ }^{\circ} \mathrm{C}$. These results show that the aggregation behaviour of bolaamphiphiles can not only be tuned by the length of the alkyl chain and the size and charge of the headgroups but also by the size difference between the two headgroups attached at one alkyl chain. When this difference becomes too large the preferred aggregation type is no longer a helical fibre but a lamellar aggregate with interdigitated chains.

\section{Acknowledgements}

This work was supported by grants from the Deutsche Forschungsgemeinschaft (Bl 182/19-3 and Do 463/4-2). The support of Dr Gerd Hause (Biocenter, Martin-Luther-University HalleWittenberg) by providing us access to the electron microscope facility is greatly appreciated. S.D. and B.D. thank Dr Thomas Markowski for his help in the synthesis.

\section{References}

1 K. Köhler, G. Förster, A. Hauser, B. Dobner, U. F. Heiser, F. Ziethe, W. Richter, F. Steiniger, M. Drechsler, H. Stettin and A. Blume, Angew. Chem., Int. Ed., 2004, 43, 245. 
2 K. Köhler, G. Förster, A. Hauser, B. Dobner, U. F. Heiser, F. Ziethe, W. Richter, F. Steiniger, M. Drechsler, H. Stettin and A. Blume, J. Am. Chem. Soc., 2004, 126, 16804.

3 K. Köhler, A. Meister, G. Förster, B. Dobner, S. Drescher, F. Ziethe, W. Richter, F. Steiniger, M. Drechsler, G. Hause and A. Blume, Soft Matter, 2006, 2, 77.

4 A. Meister and A. Blume, Curr. Opin. Colloid Interface Sci., 2007, 12, 138.

5 A. Meister, S. Drescher, I. Mey, M. Wahab, G. Graf, V. M. Garamus, G. Hause, H.-J. Mögel, A. Janshoff, B. Dobner and A. Blume, J. Phys. Chem. B, 2008, 112, 4506.

6 M. Wahab, P. Schiller, R. Schmidt and H. J. Mögel, Langmuir, 2010, 26, 2979.

7 A. Meister and A. Blume, Adv. Planar Lipid Bilayers Liposomes, 2012, 16, 93.

8 A. Blume, S. Drescher, A. Meister, G. Graf and B. Dobner, Faraday Discuss., 2013, 161, 193.

9 S. Drescher, A. Meister, G. Graf, G. Hause, A. Blume and B. Dobner, Chem.-Eur. J., 2008, 14, 6796.

10 S. Drescher, G. Graf, G. Hause, B. Dobner and A. Meister, Biophys. Chem., 2010, 150, 136.

11 S. Drescher, A. Meister, A. Blume, G. Karlsson, M. Almgren and B. Dobner, Chem.-Eur. J., 2007, 13, 5300.

12 A. Meister, S. Drescher, V. M. Garamus, G. Karlsson, G. Graf, B. Dobner and A. Blume, Langmuir, 2008, 24, 6238.

13 A. Meister, S. Drescher, G. Karlsson, G. Hause, U. Baumeister, G. Hempel, V. M. Garamus, B. Dobner and A. Blume, Soft Matter, 2010, 6, 1317.

14 G. Graf, S. Drescher, A. Meister, V. M. Garamus, B. Dobner and A. Blume, J. Colloid Interface Sci., 2013, 393, 143.

15 M. Masuda and T. Shimizu, Chem. Commun., 2001, 2442.

16 M. Masuda and T. Shimizu, Langmuir, 2004, 20, 5969.
17 J.-H. Fuhrhop and T. Wang, Chem. Rev., 2004, 104, 2901.

18 J.-H. Fuhrhop, D. Spiroski and C. Boettcher, J. Am. Chem. Soc., 1993, 115, 1600.

19 S. Drescher and B. Dobner, Synth. Commun., 2013, DOI: 10.1080/00397911.2013.825806, accepted.

20 O. Glatter, J. Appl. Crystallogr., 1977, 10, 415.

21 J. S. Pedersen, Adv. Colloid Interface Sci., 1997, 70, 171.

22 A. Meister, M. Bastrop, S. Koschoreck, V. M. Garamus, T. Sinemus, G. Hempel, S. Drescher, B. Dobner, W. Richtering, K. Huber and A. Blume, Langmuir, 2007, 23, 7715.

23 G. Graf, S. Drescher, A. Meister, B. Dobner and A. Blume, J. Phys. Chem. B, 2011, 115, 10478.

24 M. Jackson, P. I. Haris and D. Chapman, Biochim. Biophys. Acta, 1989, 998, 75.

$25 \mathrm{~J}$. W. Goodwin and R. W. Hughes, Rheology for Chemists - An Introduction, The Royal Society of Chemistry, Cambridge, 2000.

26 T. Mezger, Das Rheologie-Handbuch: Für Anwender von Rotations- und Oszillations-Rheometern, Vincentz Verlag, Hannover, 2000.

27 C. Storm, J. J. Pastore, F. C. MacKintosh, T. C. Lubensky and P. A. Janmey, Nature, 2005, 435, 191.

28 P. Terech, D. Pasquier, V. Bordas and C. Rossat, Langmuir, 2000, 16, 4485.

29 C. W. Macosko, Rheology - Principles, Measurements, and Applications, VCH Publishers, Inc., New York, 1994.

30 W. Pohle, C. Selle, W. Rettig, U. Heiser, B. Dobner and S. Wartewig, Arch. Biochem. Biophys., 2001, 396, 151.

31 A. Gliozzi, in Handbook of Nonmedical Applications of Liposomes, ed. Y. Barenholz and D. D. Lasic, CRC Press, Boca Raton, New York/London/Tokyo, 1996, vol. II, p. 329. 